\title{
Ein Leben auf Sparflamme
}

\section{Die Beratung wohnungsloser Menschen muss drei Aspekte im Blick haben: die Verbesserung der ökonomischen Basis der Ratsuchenden, die Bewältigung kritischer Lebensereignisse, die Förderung sozialer Unterstützung}

\section{Harald Ansen}

\begin{abstract}
Being without fixed adobe is only one part of the wider problem concerning emergency in connection with accommodation. Providing advice for people without accommodation (that is firmly contracted) has to watch closely the causes and it has to take the living conditions as point of reference. The wide range of questions brought forward clearly shows that this advisory work is just a variation on giving social advice.
\end{abstract}

\begin{abstract}
Manquer d'adobe n'est seulement quune partie d'un problème plus large concernant le logement d'urgence. Fournir du conseil aux personnes sans logis (par contrat) doit inclure une recherche minutieuse des causes et doit se référer aux conditions de vie. Le large panel de questions utilisé avec les personnes qui cherchent conseil en matière de logement montre clairement que ce travail de conseil n'est qu'une déclinaison d'un service de conseil social.
\end{abstract}

Prof. Dr. Harald Ansen unterrichtet Handlungsmethoden und Theorien der Sozialen Arbeit an der Hochschule für Angewandte Wissenschaften Hamburg. E-Mail harald.ansen@sp.haw-hamburg.de
Die Wohnungslosigkeit stellt nur einen Teil der Wohnungsnotfallproblematik dar. Die Beratung von Menschen obne (mietrechtlich abgesicherte) Wohnung muss sich eng an den Ursachen und den Lebensumständen der Wohnungslosen orientieren. Die thematische Breite der Wohnungslosenberatung unterstreicht, dass es sich um eine Variante der sozialen Beratung handelt.

Wohnungslosigkeit ist ein vielschichtiges Problem. Die Begriffsgeschichte vom Wanderarmen über den Nichtsesshaften und Obdachlosen zum alleinstehenden Wohnungslosen bis zum Wohnungsnotfall dokumentiert den Wandel der Sichtweisen über Wohnungslosigkeit. Während noch bis in die 1980er Jahre pathologisierende und individualisierende Ursachen in der öffentlichen und fachlichen Diskussion eine Rolle gespielt haben (vgl. Lutz 2004, Teil 1, 285), wird Wohnungslosigkeit heute vor allem auf Armut und Engpässe auf dem Wohnungsmarkt zurückgeführt.

Entgegen dem allgemeinem Wortverständnis bezieht sich Obdachlosigkeit auf Personen oder Familien, die behördlich mit einer Notlösung versorgt werden, während Wohnungslose im Freien, in Notbehelfen oder in Übernachtungsangeboten schlafen (vgl.Geißler 2006, 210). Beide Gruppen sind aber wohnungslos, da sie über keinen miet- oder eigentumsrechtlich geschützten Wohnraum verfügen.

Mit einer Veröffentlichung des Deutschen Städtetages hat sich seit 1987 die Sammelbezeichnung Wohnungsnotfälle auch in der wissenschaftlichen Betrachtung der Wohnungslosigkeit durchgesetzt. Bei Wohnungsnotfällen wird zwischen Personen unterschieden, die unmittelbar von Wohnungslosigkeit etwa wegen einer Räumungsklage bedroht sind, die ohne mietrechtlich abgesicherte Wohnung oder Wohneigentum institutionell in Wohnheimen, Notunterkünften oder bei Freunden und Bekannten vorübergehend leben, die manifest wohnungslos sind oder deren Wohnverhältnisse in Bezug auf Größe, Ausstattung oder bauliche Substanz unzumutbar sind (vgl. Busch-Geertsema/Evers/Ruhstrat 2004, 47 f.).

Die Wohnungslosenberatung richtet sich vor allem an Personen, die auf der Straße oder in Übernachtungsangeboten schlafen sowie an institutionell untergebrachte wohnungslose Menschen. Diese Zielgruppe ist auf umfassende Hilfen angewiesen, die weit über die reine Unterbringung hinausgehen. Die Wohnungslosenberatung in dem hier gewählten Zuschnitt kann in ambulanten Beratungsstellen, in Wohneinrichtungen und in der aufsuchenden Sozialarbeit eingesetzt werden. Sie ist auch für Fachstellen relevant, die Beratungs- und Unterstützungsangebote für die gesamte Palette von Wohnungsnotfällen vorhalten.

\section{Ursachen der Wohnungslosigkeit}

Eine bundeseinheitliche Wohnungslosenstatistik wird nicht geführt. Angaben über Wohnungslosigkeit basieren auf Schätzungen, die in erster Linie von der Bundesarbeitsgemeinschaft Wohnungslosenhilfe durchgeführt werden. Danach leben in der Bundesrepublik Deutschland (Stand: 2004) etwa 345.000 Wohnungslose, darunter rund 148.000 in Mehrpersonenhaushalten, 144.000 in Einpersonenhaushalten und etwa 20.000 ohne jede Unterkunft auf der Straße (vgl. http://www.bag-wohnungslosenhilfe.de am 16.1.2007).

Es wäre verfehlt, wollte man die Ursachen der Wohnungslosigkeit vorwiegend auf individuelle Faktoren reduzieren. Versorgungsengpässe mit bezahlbarem Wohnraum auf dem Wohnungsmarkt sind, von regionalen Schwankungen abgesehen, primär für die verbreitete Woh- 
nungslosigkeit verantwortlich. Wenn dann noch individuelle Ursachen wie Überschuldung oder Verlust des Arbeitsplatzes hinzu kommen, steigt das Risiko der Wohnungslosigkeit.

Das Ziel der Wohnungslosenberatung besteht darin, Wohnungslose in eigenen Wohnraum zu vermitteln. Die formalen Schritte zu diesem Ziel sind überschaubar und nicht besonders komplex. Allerdings ist in vielen Fällen eine isolierte Bearbeitung der Wohnungslosigkeit angesichts der vielfältigen Ursachen nicht möglich. Soziale und persönliche Barrieren auf dem Weg in die eigene Wohnung stehen im Mittelpunkt der Beratung, sie sind häufig auch ursächlich mitverantwortlich für den Verlust der Wohnung. Insofern ist die Wohnungslosenberatung per se soziale Beratung mit dem besonderen Fokus der Überwindung von Wohnungslosigkeit.

$\mathrm{Zu}$ den häufigsten Ursachen für den Verlust der bisherigen Wohnung zählen mietschuldenbedingte Kündigungen und Zwangsräumungen. Wohnungslosigkeit entsteht auch dann, wenn Menschen aus Justizvollzugsanstalten, langfristigen therapeutischen Behandlungen, Frauenhäusern oder anderen Einrichtungen entlassen werden und vorher keine Wohnung vorhanden war. Eine weitere Ursache liegt in Zuwanderung und Flucht aus der bisherigen Heimat (vgl. Hradil 2005, 328). Eine wesentliche Ursache für den Verlust der Wohnung ist finanzielle Armut bei Arbeitslosigkeit, Krankheit oder bei geringem Verdienst.

In diesem Zusammenhang auftretende Mietschulen, häufig in Verbindung mit weiteren Schulden, führen in vielen Fällen trotz des vorhandenen Mieterschutzes aufgrund fehlender Gegenwehr der Betroffenen zum Verlust der Wohnung (vgl. Gerull 2002, 89 f.). Die primäre Aufgabe der Wohnungslosenberatung besteht darin, die materielle Armut der Ratsuchenden durch die Erschließung von Sozialleistungen und die Unterstützung bei der Arbeitssuche abzubauen. Die Ausrichtung auf die materielle Armut reicht häufig aber nicht aus, um Wohnungslosigkeit zu überwinden.

Die teilweise geringe Bereitschaft oder Fähigkeit, Hilfen zur Sicherung der Wohnung zu beanspruchen, hat persönliche und soziale Gründe. Zu den persönlichen Gründen zählen u. a. Bildungsdefizite. Viele Betroffene kennen ihre sozialen
Rechte bei Mietschulden und das Hilfesystem nicht. Sie verhalten sich nicht »verwaltungskonform " und verlieren schon deshalb ihre Wohnung. Hinzu kommen kritische Lebensereignisse wie die Trennung vom Partner, der Verlust des Arbeitsplatzes oder eine schwere Erkrankung. Betroffene reagieren häufiger nicht auf Hilfeangebote, sie ziehen sich zurück, resignieren und betrachten den Verlust der Wohnung als unabwendbares Schicksal oder persönliches Scheitern. Wer in diese Verfassung gerät, zeigt wenig Initiative, die Wohnungslosigkeit zu überwinden. Die Wohnungslosenberatung steht hier vor der Aufgabe, Menschen einerseits über Unterstützungsmöglichkeiten aufzuklären und entsprechende Handlungskompetenzen aufzubauen und sie andererseits in der Überwindung kritischer Erfahrungen zu unterstützen und sie zu motivieren, ihre Lebensumstände zu verändern. Ohne diese Vorarbeit bleiben die Bemühungen um eine Wohnung durch die Beratungsstelle tendenziell erfolglos.

Soziale Gründe für Wohnungslosigkeit liegen in fehlender sozialer Unterstützung im Alltag der Betroffenen, die in Krisenzeiten dann leichter aus der Bahn geworfen werden. Hinzu kommen Stigmatisierungen von Arbeitslosen, ehemaligen Strafgefangenen oder psychisch kranken Menschen, die schon deshalb geringere Chancen haben, eine Wohnung zu finden. Die ökonomische und soziale Ausgrenzung trägt dazu bei, dass persönliche Belastungen eskalieren und Notlagen wie die Wohnungslosigkeit schnell chronisch verlaufen. In der Wohnungslosenberatung fällt deshalb der Förderung von sozialer Unterstützung im Alltag der Betroffenen eine prominente Rolle zu. Erst wenn die mehrdimensionalen Ursachen der Wohnungslosigkeit in der Beratung aufgegriffen werden, können Drehtüreffekte in der Wohnungslosenhilfe vermieden und mit den Ratsuchenden langfristig tragfähige Lösungen erreicht werden.

Die Ursachen der Wohnungslosigkeit verweisen auf eine Verlustspirale in der Ressourcenausstattung (vgl. Nestmann 2004, 75). So geht beispielsweise der Verlust des Arbeitsplatzes mit Einkommenseinbußen und häufig auch mit dem Verlust sozialer Kontakte und vermehrten familiären Spannungen einher. Diese Kumulation forciert soziale Ausgren- zungsprozesse und erhöht das Risiko des Wohnungsverlustes.

Bezogen auf die Ursachen der Wohnungslosigkeit sind in der Beratung drei Bereiche besonders wichtig. Zum einen geht es darum, die ökonomische Basis der Ratsuchenden zu verbessern, zum zweiten bei Bedarf um die Bewältigung kritischer Ereignisse, die mit zum Verlust der Wohnung beigetragen haben, und drittens um die Förderung sozialer Unterstützung, die für die Stabilisierung von Ratsuchenden relevant ist. Hinter diesen formalen Kategorien verbergen sich Lebensbelastungen, die einer einfachen Lösung im Weg stehen.

\section{Alltägliche Belastungen bei Wohnungslosigkeit}

Ohne Wohnung leben, so LudwigMayerhofer u.a. (2001, 273 f.), beinhaltet Gefahren für die körperliche und seelische Gesundheit, Betroffene sind Stigmatisierungen ausgesetzt und von Gewalt bedroht, das Zusammenleben mit Partnern und einer Familie ist erschwert oder unmöglich und die Bewältigung des Alltags in Bereichen wie Ernährung oder Körperpflege ist extrem anstrengend. Ein Leben ohne Wohnung grenzt aus, Teilhabe an den Errungenschaften der Gesellschaft wird systematisch verhindert. Die negativen Konsequenzen treten vor allem bei einem langfristigen Verlauf auf. Etwa ein Drittel der alleinstehenden Wohnungslosen lebt nach aktuellen Erhebungen schon drei Jahre und ein Fünftel bereits seit rund fünf Jahren auf der Straße (vgl. Hradil 2005, 330). Je länger die Wohnungslosigkeit anhält, desto größer ist die Gefahr, dass Menschen resignieren und ihre anfänglichen Bemühungen um eine neue Wohnung einstellen.

Die Beurteilung der Lebensumstände durch die Wohnungslosen selbst ist heterogen. In ihrem Reportageband »Die Dritte Haut « (2006) hat Katrin Panier rund 20 Geschichten über Wohnungslosigkeit in Deutschland zusammengestellt. Mit der dritten Haut bezieht sich Panier auf die drei Häute nach Friedensreich Hundertwasser. Der Maler hat die natürliche Haut von der Kleidung als der zweiten Haut und der Wohnung als der dritten Haut unterschieden. Die drei Häute stehen für die Verletzlichkeit des Menschen. 


\section{»Das Maß an Zufriedenheit ist beachtlich«}

In einer eigenen Erhebung im Sommer 2006 wurden die Überlebensstrategien Wohnungsloser in Hamburg in zehn narrativen Interviews beleuchtet. Die Befragten waren zwischen 20 und 50 Jahre alt, wobei ältere Interviewpartner dominierten. Alle Befragten machen zwischen drei und fünf Jahren »Platte ", die Mehrzahl lebt länger als fünf Jahre in Hamburg. Sie halten sich überwiegend in der Innenstadt auf. Eine geschlechtsspezifische Differenzierung der Aussagen wurde nicht durchgeführt.

Die Auswahl der Interviewpartner erfolgte nach dem Zufallsprinzip in einer Tagesaufenthaltsstätte. Bei den Interviews ging es nicht um eine repräsentative Erhebung, die mit qualitativen Forschungsmethoden ohnehin kaum möglich ist, sondern um Einblicke in die Lebenswelt der Betroffenen aus ihrer Perspektive. Im folgenden werden ausgewählte Befunde erläutert, die für die Wohnungslosenberatung relevant erscheinen.

Die Befragten haben die Übernachtungsmöglichkeiten der Stadt Hamburg, abgesehen vom Winternotprogramm, durchgängig negativ beurteilt. Mangelnde Hygiene, zu viele Menschen auf engem Raum, zu geringe Kontrolle und Angst vor Diebstählen waren dafür ausschlaggebend. Das Niveau der Übernachtungsangebote wird insgesamt als zu niedrig eingeschätzt. Für die Wohnungslosenberatung zeigt dieser Punkt, dass es nicht reicht, auf Hilfemöglichkeiten zu verweisen, die für Ratsuchende inakzeptabel sind. Legitime Ansprüche an einen Mindeststandard sollten in der Beratung ebenso berücksichtigt werden wie individuelle Bedürfnisse, die für die Inanspruchnahme einer Hilfe bedeutsam sind. Ein formales Vorgehen bei der Organisation einer Übernachtungshilfe greift viel zu kurz.

Die Interviewpartner beziehen in der Regel Leistungen nach dem SGB II oder ein in der Höhe analoges Einkommen. Die meisten gaben an, dass sie mit ihrem Einkommen zufrieden seien. Sie nutzen u. a. Kleiderausgabestellen oder kostenlose Mahlzeiten und verfügen teilweise über geringe Zuverdienste. Aus sozialwissenschaftlicher Sicht wird moniert, dass die Regelleistung nach SGB II das soziokulturelle Existenzminimum deutlich unterschreitet. Für die Betroffenen scheint diese Einschätzung nicht zuzutreffen. Sie haben sich mit ihren Möglichkeiten arrangiert und führen ein »Leben auf Sparflamme«. Die Gewöhnung an materielle Armut und die Verzichtsbereitschaft sollten in der Wohnungslosenberatung aufgegriffen werden. Ratsuchende sollten offensiv über Sozialleistungen aufgeklärt und im Antragsverfahren unterstützt werden.

Alle Gesprächspartner gaben an, dass sie mit ihren Beziehungen in der Wohnungslosenszene und auch außerhalb zufrieden sind. Befragte, die über ein sehr kleines Netz verfügen, wünschten sich keine andere Situation. Das Maß an Zufriedenheit ist beachtlich. Es widerspricht der Außenwahrnehmung von sozialer Ausgrenzung und fehlender sozialer Unterstützung in extremer Armut. Wie bei anderen Antworten könnte man annehmen, dass sich die Befragten an ihre Lebensumstände gewöhnt haben und weitergehende Wünsche nicht mehr zulassen. In der Wohnungslosenberatung kommt es aber darauf an, an die subjektiven Sichtweisen der Ratsuchenden anzuknüpfen, um einen Zugang zu finden und vor al- lem um mit ihnen Lösungen zu erarbeiten, die ihren Relevanzkriterien entsprechen. Im Sinne der lebensweltorientierten Sozialen Arbeit (vgl. Thiersch 2005) ist es für die Beratung wichtig, sehr behutsam mit den Alltagsinterpretationen von Ratsuchenden umzugehen und möglicherweise dahinterliegende Motive und Bedürfnisse aufzudecken. Entfremdende Lösungen führen zum vorzeitigen Abbruch der Beratung oder zu nicht belastbaren Hilfeangeboten.

In Bezug auf Erkrankungen berichteten alle interviewten Personen, dass Wohnungslosigkeit nicht zu einer erhöhten Krankheitsanfälligkeit oder speziellen Erkrankungen führt. Jeder müsse auf sich achten. Die älteren Befragten gaben allerdings an, dass sie spüren, wie ihre Kräfte nachlassen und sie längerfristig eine Änderung ihrer Lebensumstände anstreben. Diese Selbsteinschätzung steht konträr zu den sozialmedizinischen und sozialepidemiologischen Befunden, wonach Wohnungslose in nahezu allen Krankheitsbereichen vulnerabler als andere Bevölkerungsgruppen sind und auch eine um Jahre geringere Lebenserwartung als der Bevölkerungsdurchschnitt haben (vgl. stv. Trabert 2005, 161 f.).

Für die Selbsteinschätzung der Betroffenen mag eine ausgeprägte Symptomtoleranz eine wichtige Rolle spielen. Im übrigen erkennen auch Menschen in anderen prekären Lebenslagen häufig nicht Zusammenhänge zwischen ihrem Lebensstil einschließlich ihren alltäglichen Belastungen und auftretenden Erkrankungen. Sozialmedizinische Themen wie die Versorgungsqualität im medizinischen System, Zugänge zu Behandlungsleistungen für Wohnungslose und Änderungen belastender Lebensstile können in der Wohnungslosenberatung nicht ausgeklammert werden. Die vielerorts etablierten medizinischen Elementarhilfen können nur ein Baustein für eine angemessene Versorgung sein. Aus der Sicht der Interviewten werden diese Angebote in Hamburg als sehr gut und hilfreich beurteilt, wobei von vielen angemerkt wird, dass diese Hilfen vor Ort bei komplexen Erkrankungen und aufwendigen Untersuchungen nicht ausreichen.

Noch ein letzter Aspekte der Interviewauswertung: Mittelfristig streben alle Befragten eine Änderung ihrer Situation an, eilig hat es aber niemand. Offenbar erreichen sie die formellen und informellen Hilfen Wohnungslose ausreichend und flankieren in ihrem sehr belasteten Alltag. Die Befragten betonten besonders die niedrigschwelligen Hilfen und die Basisversorgung. Niemand müsse hungern oder ungepflegt herumlaufen. Die vordergründig geringe Änderungsmotivation beeinflusst den Beratungsprozess. Angesichts der häufig kumulativen Ursachen für Wohnungslosigkeit, der belastenden aktuellen Lebensumstände und der langen Dauer der Wohnungslosigkeit überrascht die zögerliche Haltung der Betroffenen nicht. Sie haben ihr Tempo entwickelt, das in der Beratung zu berücksichtigen ist. Kurzfristige Erfolge sind häufig nicht haltbar. Die Rückkehr in ein Leben außerhalb der Wohnungslosigkeit braucht Zeit, die im Hilfeprozess eingeräumt werden sollte. Ein zu hohes Tempo führt bei den Betroffenen zu Rückschlägen, die ihre Änderungsbereitschaft zusätzlich unterminieren.

Harald Ansen 
Wohnungslose berichten einerseits über die Einsamkeit und Trostlosigkeit ihres Alltags, über die Bedrohungen und Belastungen, andererseits äußern Wohnungslose, dass sie die Freiheit ihres Lebens genießen und das differenzierte Hilfeangebot für ausreichend erachten. Für wieder andere, vor allem jüngere Menschen, ist die Szene ein Ersatz für eine fehlende Familie und ein nicht vorhandenes schützendes Milieu außerhalb der Wohnungslosentreffpunkte. Angesichts der objektiven Belastungen bei Wohnungslosigkeit ist die Gefahr groß, dass die persönlichen Aussagen der Betroffenen über ihre Freiheit und Zufriedenheit mit ihrem gegenwärtigen Leben von den Sozialarbeitern in den Beratungsstellen als mentale Anpassung an eine ausweglose Lage interpretiert werden. So einfach sollte man aber die subjektiven Äußerungen nicht entkräften (vgl. nebenstehenden Kasten »Das Maß an Zufriedenheit ist beachtlich «).

\section{Interventionen bei Wohnungslosigkeit}

$\mathrm{Zu}$ fragen ist, ob die Berücksichtigung der Ursachen und der Lebenslagemerkmale der Ratsuchenden in der Wohnungslosenberatung mit dem sozialhilferechtlichen Auftrag zu vereinbaren ist. Sowohl die Charakterisierung des Personenkreises, bei denen besondere Lebensverhältnisse mit sozialen Schwierigkeiten verbunden sind ( $\mathbb{S} 67$ SGB XII) als auch der Hinweis in $\ 68$ Abs. 1 SGB XII, wonach die Hilfe alle notwendigen Maßnahmen beinhaltet, weisen darauf hin, dass eine umfassende Beratung wohnungsloser Menschen im Sinne des SGB XII gerechtfertigt ist. Ein Leben auf der Straße oder in Notbehelfen stellt im Sozialhilferecht eine Interventionsschwelle dar. Um den Besonderheiten des Einzelfalls entsprechen zu können, wurde der notwendige Hilfebedarf nicht pauschal geregelt, sondern in das Ermessen des Sozialhilfeträgers gestellt. Wohnungslose, die Hilfe nach dem SGB XII beanspruchen, dürfen allerdings nicht genötigt werden, sich sozial anzupassen. Die Unterstützung erfolgt situationsbezogen und nicht persönlichkeitsbildend, wie nach dem SGB VIII (vgl. Sozialrechtsberatung 2005, 612 f.).

In den Bereich der Interventionen zur Sicherung der materiellen Lebensgrundla- gen gehören Informationen der Ratsuchenden über und Hilfen zur Erschließung existenzsichernder Sozialleistungen nach SGB II und XII und, soweit ein Anspruch besteht, auch vorgelagerter Sozialleistungsansprüche. Für die Umsetzung dieses Beratungsziels sind sozialadministrative Kompetenzen erforderlich, die umfassende Gesetzeskenntnisse zur sozialen Sicherung ebenso voraussetzen wie Verwaltungskompetenzen, um die behördlichen Zuständigkeiten zu ermitteln und die Ratsuchenden im Antragsverfahren und bei der Rechtsdurchsetzung zu unterstützen (vgl. Ansen 2006, 169 f.). Zur Sicherung der materiellen Lebensgrundlagen zählen Informationen über Kleiderausgabestellen, Mahlzeitendienste und Übernachtungsmöglichkeiten. Die dagogisch betreute Wohnformen. Neben der Vermittlung dieser Hilfen werden im Rahmen der Wohnungslosenberatung auch erforderliche Unterstützungsmaßnahmen aus anderen Bereichen erschlossen. Hierzu zählen u. a. die Schuldnerberatung und medizinische Hilfen. Die Wohnungslosenhilfe sollte es vermeiden, immer mehr Unterstützungsangebote in ihr System zu integrieren, denn damit wird die Ausgrenzung ihrer Zielgruppe zementiert. Beratungen, Behandlungen und Therapien sollten dort durchgeführt werden, wo auch andere beraten, behandelt und therapiert werden (vgl. Lutz 2004, 329 f.). Dieser Anspruch der Wohnungslosenberatung setzt eine umfassende Kooperation mit anderen Hilfestellen voraus.

\section{»In vielen Fällen ist eine isolierte Bearbeitung der Wobnungslosigkeit angesichts der vielfältigen Ursachen gar nicht möglich «}

Wohnungslosenhilfe umfasst behördliche und behördlich (mit-) finanzierte Angebote der Wohlfahrtsverbände, aber auch Hilfen durch Freiwillige und im Rahmen des bürgerschaftlichen Engagements. In der Wohnungslosenberatung sind umfassende Kenntnisse des regionalen Versorgungssystems ein unverzichtbares Handlungswissen. Die materiellen Lebensgrundlagen können in vielen Fällen nur durch eine Kombination unterschiedlicher Hilfequellen garantiert werden.

Interventionen zur Förderung der sozialen Unterstützung beziehen sich in der Wohnungslosenberatung vor allem auf formelle soziale Netze. Zunächst müssen Ratsuchende über die ambulanten und stationären Angebote der Wohnungslosenhilfe aufgeklärt werden. Mit diesem Wissen fällt es Ratsuchenden leichter, ihre Unterstützungsbedürfnisse zu artikulieren. Zu den Regelangeboten der Wohnungslosenhilfe zählen beispielsweise Beratungsstellen, die Einrichtung einer für die Bewilligung von Sozialleistungen unverzichtbaren Erreichbarkeitsadresse in Beratungsstellen oder Tagesaufenthalten, Hilfen im Alltag wie Körperpflege, Aufenthalt oder Ernährung, Übernachtungsangebote und unterschiedliche sozialpä-
Interventionen zur Förderung der persönlichen Bewältigungsmöglichkeiten zielen nicht darauf, Ratsuchende an durch wen auch immer definierte Normalitätsvorstellungen anzupassen. Vielmehr geht es darum, Ratsuchende dabei zu unterstützen, selbstbestimmt zu leben (vgl. ebd.). Persönliche Hilfen in der Wohnungslosenberatung können so breit gestreute Formen wie Krisenintervention, Wissensvermittlung, Training alltagsbezogener Kommunikation oder die Förderung der Veränderungsmotivation umfassen. Im Vordergrund steht die Vermittlung alltagspraktischer Kompetenzen, die durch ein längeres Leben auf der Straße verschüttet werden oder auch verloren gehen.

Erst die Kombination von materiellen, sozialen und persönlichen Hilfen führt bei Ratsuchenden mit komplexen Problemen zum Erfolg. Die persönliche Unterstützung zur Überwindung von Wohnungslosigkeit wird in den Entwürfen zur Weiterentwicklung der Wohnungslosenhilfe teilweise vernachlässigt. Besonders fällt das in den so genannten Fachstellenkonzepten auf, die sich um die Wohnungssicherung, die Wohnungsversorgung, die öffentlich-rechtliche Unterbrin- 
gung und das Sozialmanagement kümmern sollen. Wohnungslosigkeit ist, wie die Ausführungen über die Ursachen und die Lebensumstände gezeigt haben, nicht nur ein strukturelles Problem. Die Wohnungslosenberatung sollte ihre mehrdimensionale Ausrichtung in den genannten Interventionsbereichen vertiefen, um Ratsuchenden eine geeignetes Hilfepaket anbieten zu können.

\section{Ausblick}

Die Wohnungslosenberatung als ein Teil des Hilfesystems für Wohnungslose zählt zum ambulanten Bereich. Die Abgrenzung ambulanter, teilstationärer und stationärer Hilfeangebote überzeugt aber immer weniger. Specht-Kittler (vgl. 2005) schlägt vor, die bisherige Abgrenzung zu Gunsten eines Hilfesystems zu überwinden, in dem unterschiedliche Hilfebedarfe und Hilfeintensitäten sowie die Hilfedauer ausschlaggebend sind. Für die Wohnungslosenberatung könnte das bedeuten, dass sie mehr als bisher Teil einer organisatorischen Einheit ist und mit anderen Formen der Hilfe kombiniert wird. Diese Reorganisation könnte die Wirksamkeit der Beratung erhöhen.

Die Wohnungslosenberatung ist an einer Nahtstelle des Sozialstaates tätig, an

der deutlich wird, inwieweit es gelingt, Menschen in besonders benachteiligten Lebenslagen erforderliche Hilfen zur Verfügung zu stellen. In der Wohnungslosenberatung werden Versorgungslücken und eine unzureichende Sozialhilfepraxis erkennbar.

Die Aufgabe der Wohnungslosenberatung besteht darin, an einer Optimierung der sozialen Infrastruktur für Wohnungslose mitzuarbeiten und politische Initiativen zur Verbesserung der Rahmenbedingungen des Hilfesystems zu unterstützen. Diese nicht unmittelbar auf Ratsuchenden bezogenen Aktivitäten sollten einen ausreichenden Platz in der Weiterentwicklung der Wohnungslosenberatung einnehmen. Hier wäre es sinnvoll, Betroffene einzubinden und - wie man heute sagt - zu aktivieren.

\section{Literatur}

Ansen, Harald: Soziale Beratung bei Armut. München 2006.

Busch-Geertsema/Evers, Jürgen/Ruhstrat, Ekke-Ulf: Mehr als ein Begriff. In: Wohnungslos 2/2004, 45-49.

Geißler, Rainer: Die Sozialstruktur Deutschlands. Wiesbaden 2006.

Gerull, Susanne: Aufsuchende Hilfen in der Wohnungssicherung. In: Soziale Arbeit, 3/2002, 89-96.

Handbuch Sozialrechtsberatung. Baden-Baden 2005.

Hradil, Stefan: Soziale Ungleichheit in Deutschland. Wiesbaden 2005.

Ludwig-Mayerhofer, Wolfgang u.a.: Von der Armut, ohne Wohnung zu leben. In: Barlösius, Eva/Ludwig-Mayerhofer, Wolfgang (Hg.): Die Armut der Gesellschaft. Opladen 2001, 263-293.

Lutz, Ronald: Vaganten, Wanderer, Nichtsesshafte, Wohnungslose. Teil 1. In: Soziale Arbeit 8/2004, 282-289.
Lutz, Ronald: Vaganten, Wanderer, Nichtsesshafte, Wohnungslose. Teil 2. In: Soziale Arbeit 9/2004, 329-333.

Nestmann, Frank: Ressourcenarbeit. In: Grundwald, Klaus/Thiersch, Hans (Hg.): Praxis Lebensweltorientierter Sozialer Arbeit. Weinheim, München 2004, 69-87.

Panier, Katrin: Die dritte Haut. Berlin 2006.

Specht-Kittler, Thomas: Die Zukunft der Wohnungslosenhilfe. In: Wohnungslos 2/05, 49-56.

Thiersch, Hans: Lebensweltorientierte Soziale Arbeit. Weinheim, München 2005.

Trabert, Gerhard: Gesundheitsarbeit in der Wohnungslosenhilfe. In: Ortmann, Karlheinz/Waller, Heiko (Hg.): Gesundheitsbezogene Soziale Arbeit. Hohengehren 2005, 161-178.

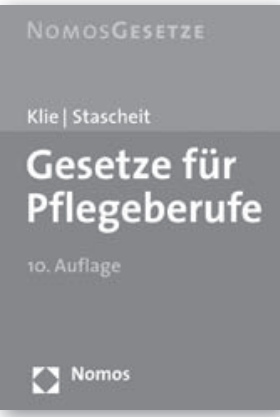

Gesetze für Pflegeberufe

Von Prof. Dr. Thomas Klie, FH Freiburg und Prof. Ulrich Stascheit, FH Frankfurt/M. 10. Auflage 2007, ca. 1.200 S., brosch., ca. 19,- $€$, ISBN 978-3-8329-2475-1

Erscheint Mai 2007

Vertiefte Rechtskenntnisse im Bereich der ambulanten und stationären Pflege, im Berufs- und Tarifrecht, im Haftungs- und Strafrecht und insbesondere im Recht der Kranken- und Pflegeversicherung gewinnen für Angehörige der Pflegeberufe, Auszubildende und Studierende der Fachhochschulen zunehmend an Bedeutung.

Die Sammlung fasst die erforderlichen Rechtsgrundlagen jetzt schon in 10. Auflage topaktuell und preiswert zusammen.

\section{Nomos}

